\title{
Estudio tafonómico del sitio arqueológico Yegua Quemada 3, Holoceno medio, Patagonia meridional
}

\section{Taphonomical analysis of the mid-Holocene Yegua Quemada 3 archaeological site (Southern Patagonia)}

\author{
DANIELA S. CAÑETE MASTRÁNGELO ${ }^{1}$, PATRICIA A. LOBBIA² \& A. SEBASTIÁN MUÑOZ² \\ ${ }^{1}$ CONICET/FFyL-UBA/Instituto Nacional de Antropología y Pensamiento Latinoamericano. 3 de Febrero 1370/78, CABA. \\ Argentina. \\ danielasol.cm@gmail.com \\ ${ }^{2}$ Laboratorio de Zooarqueología y Tafonomía de Zonas Áridas. IDACOR_CONICET/Universidad Nacional de Córdoba. Av. \\ H. Yrigoyen 174, (5000) Córdoba. Argentina. \\ smunoz@conicet.gov.ar, patalobbia@gmail.com
}

(Received 4 June 2018; Revised 29 August 2018; Accepted 24 September 2018)

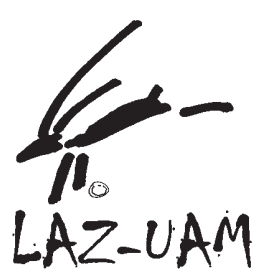

En este trabajo se presentan los resultados del análisis tafonómico efectuado en el depósito Yegua Quemada 3, Parque Nacional Monte León, con una ubicación cronológica correspondiente al Holoceno medio (5360 \pm 20 años AP). Los resultados muestran que el material arqueomalacológico se encuentra en un proceso de degradación avanzado, resultado de períodos de exposición, indicados por la pérdida de color, pero también de enterramiento, indicado por la pérdida de las capas externas y la dureza de las valvas. Los restos de vertebrados muestran asimismo la importancia que los procesos bioestratinómicos y diagenéticos tuvieron en la historia depositacional del conjunto. Las superficies óseas están afectadas de manera homogénea, lo que indica exposición a condiciones subaéreas. El conjunto artefactual lítico presenta evidencias de haber pasado por períodos de exposición subaérea, evidenciado por la abrasión eólica y períodos de enterramiento, mostrado por el desarrollo de un revestimiento de carbonato. El conjunto de evidencias considerado y las variables tafonómicas analizadas permiten concluir que el depósito YQ3 brinda información adecuada para discutir la ocupación humana al sur del río Santa Cruz en un bloque temporal, el Holoceno medio, período para el cual se cuenta con limitada información.

PALABRAS CLAVE: TAFONOMÍA, PROCESOS DE FORMACIÓN DE SITIOS, CAZADORES-RECOLECTORES, PATAGONIA, HOLOCENO MEDIO

\begin{abstract}
This paper presents the results of the taphonomic analysis carried out in the Yegua Quemada 3 (YQ3) archaeological deposit, Monte León National Park, dated back to the mid Holocene $(5360 \pm 20$ years BP). The results show that the archaeomalacological materials are in a process of advanced degradation, being the result of exposure, indicated by the loss of color, but also of burial, indicated by the loss of the outer layers and hardness of shells. Vertebrate remains also show the importance that biostratinomic and diagenetic processes had in the site depositional history. Bone surfaces are affected in a homogeneous way, indicating exposure to subaerial conditions. The lithic artefactual assemblage displays evidence of having gone through periods of subaerial exposure, evidenced by wind abrasion, as well as burial periods, shown by the development of a carbonate skin. The set of evidences considered and the taphonomic variables analyzed here allows to conclude that YQ3 deposit provides adequate data to discuss the human occupation south of the Santa Cruz River in the mid Holocene.
\end{abstract}

KEY WORDS: TAPHONOMY, SITE FORMATION PROCESSES, HUNTER-GATHERERS, PATAGONIA, MID HOLOCENE

http://doi.org/10.15366/archaeofauna2019.28.003 


\section{INTRODUCCIÓN}

El registro arqueológico del Holoceno medio de la costa atlántica continental de Patagonia está formado por depósitos con resoluciones muy distintas, por lo que disponer de análisis tafonómicos es una herramienta clave para su interpretación y análisis comparativo (Caracotche et al., 2017). Aunque el registro arqueológico costero es abundante en el bloque temporal comprendido por los últimos 3000 años radiocarbónicos, éste resulta mucho más fragmentario cuando se trata de momentos más antiguos (ver Castro et al., 2007: cuadros 2 a 4). La situación para el área abarcada por el actual Parque Nacional Monte León (PNML), al sur del río Santa Cruz, no escapa a esta tendencia general (Caracotche et al., 2005; Muñoz et al., 2009), por lo que la evaluación detallada de la información disponible en el bloque temporal anterior a 3000 años AP es un requisito imprescindible para profundizar las discusiones en torno al uso humano de las zonas actualmente costeras de este sector de Patagonia meridional.

En el PNML se han identificado pequeños depósitos malacológicos lenticulares asociados a escasos restos líticos y óseos pertenecientes al Holoceno medio, que son los sitios CCH1 (5550 +/- 90 años AP) y CCH3 (4160+/- 80 años AP) (Caracotche et al., 2005; Muñoz et al., 2009) y solo un depósito con una composición más diversa y compleja, el sitio Yegua Quemada 3 (5360 \pm 20 -UGAMS 11762-, ver Caracotche et al., 2017). Aquí presentamos una evaluación tafonómica sobre este último depósito.

El Parque Nacional Monte León está ubicado sobre la costa atlántica, en la planicie que se extiende entre el río Santa Cruz por el norte y el río Coyle por el sur. El Parque comprende dos áreas ecológicas de la Estepa Patagónica: la de la Meseta Central y la del Matorral de Mata Negra. La primera, que es la de interés para esta investigación, está representada en el área costera, de cañadones y de lomadas intermedias. Se trata de una estepa de arbustos enanos, rastreros, de muy baja cobertura vegetal (entre 20 y $30 \%$ ), con arbustos de porte mayor que siguen las líneas de escorrentía y grandes áreas cubiertas de pavimento de erosión (muy frecuentes en Monte León) en un relieve plano, de planicies aluviales de rodados patagónicos y mesetas sedimentarias. El clima predominante de esta área es el Frío Árido de Meseta, con una tempera- tura promedio de 10 a $8^{\circ} \mathrm{C}$. Las precipitaciones son inferiores a $150 \mathrm{~mm}$ y presentan una concentración invernal (Oliva et al., 2006; Cruz et al., 2011).

\section{EL DEPÓSITO}

El conjunto de materiales recuperados en Yegua Quemada 3 (YQ3) es el más diverso de los procedentes de la costa del PNML con datación correspondiente al Holoceno medio (Figura 1). El análisis de las distintas clases de evidencia recuperadas en éste depósito ha sido desarrollada previamente (Caracotche et al., 2017), por lo que en este trabajo lo que efectuamos es un análisis tafonómico del conjunto total recuperado. El sitio arqueológico consiste en un relicto de una lente de conchero expuesto, que se encuentra a $1,10 \mathrm{~m}$ por debajo del suelo actual, en una cicatriz de erosión ubicada en el frente de un acantilado. El depósito, está ubicado $8 \mathrm{~m}$ sobre el nivel de la línea de costa actual y forma parte de un médano compactado localizado en los niveles aterrazados de la costa sur del Parque Nacional (Figura 2). El acantilado presenta una topografía descendente, con pendiente abrupta hacia

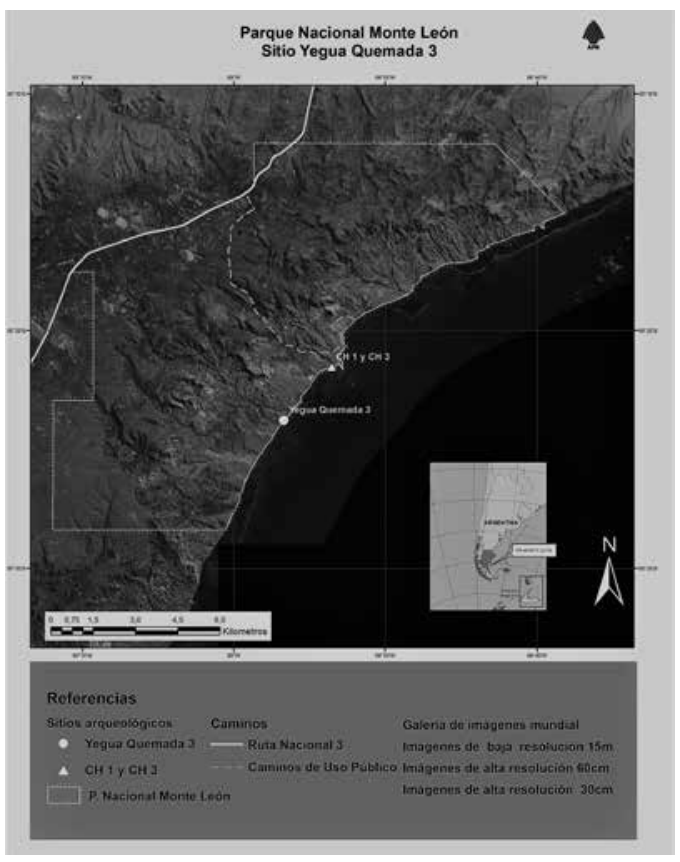

FIGURA 1

Localización del sitio arqueológico YQ3 en el Parque Nacional Monte León (imagen tomada de Caracotche et al., 2017: fig. 1). 


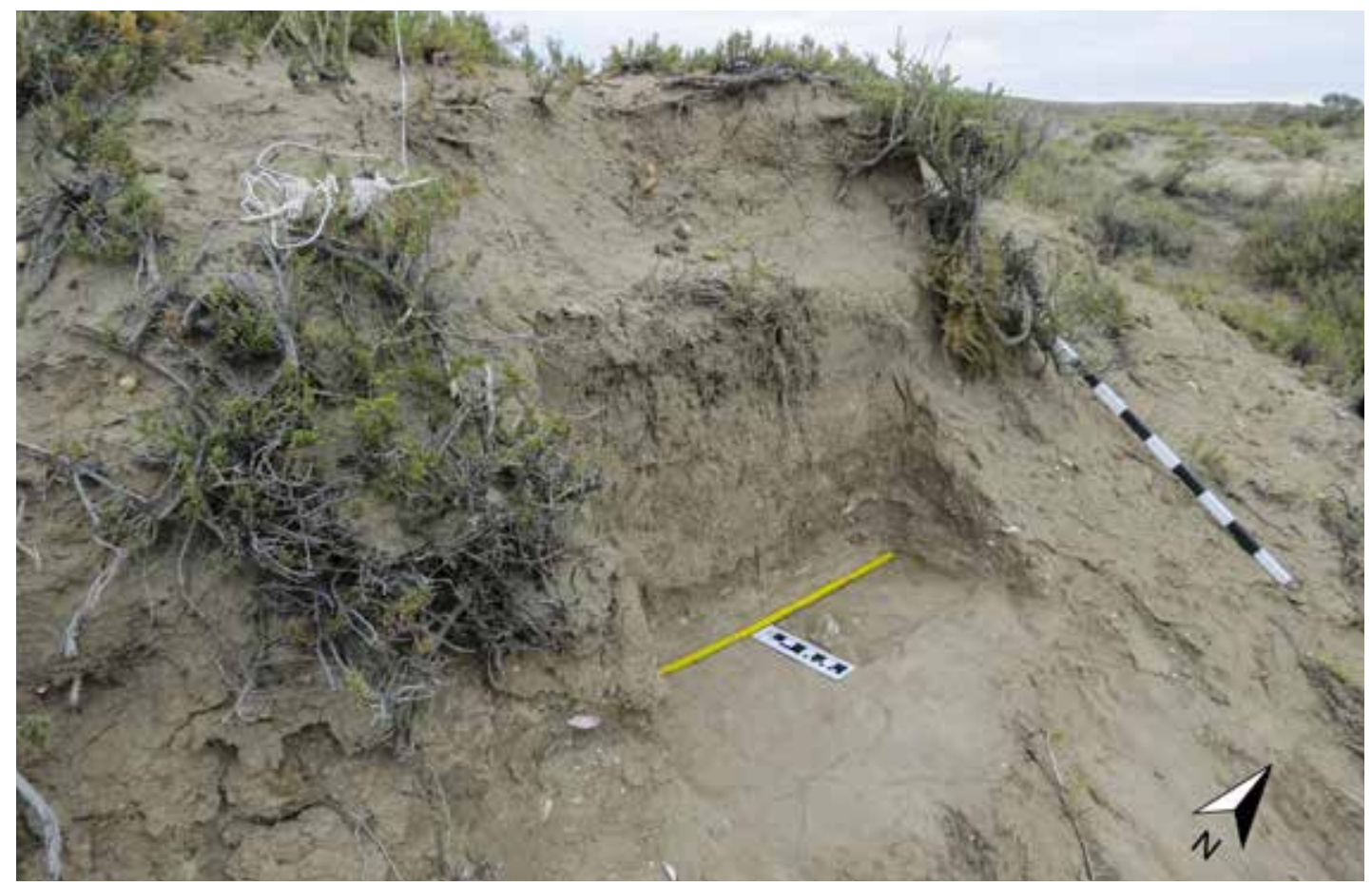

FIGURA 2

Vista general del sitio YQ3 y cuadrícula de excavación.

la playa y está cubierto por vegetación arbustiva, con cantos rodados en la base. La pendiente hacia la playa oscila entre $50^{\circ}$ y $60^{\circ}$, presentando la ladera sectores de arena suelta y algunos arbustos con restos arqueológicos dispersos en superficie. Estos materiales son el resultado de la circulación de agua que los transportó por gravedad hacia las playas de rodados, por lo que puede estimarse que en el pasado el depósito contó con una mayor extensión, habiendo desaparecido parte de su volumen por la erosión y la formación de escorrentías.

El depósito presenta una matriz homogénea que cambia de composición a una profundidad de 1,37 m. A partir de este nivel el cambio de sedimento registrado se ve acompañado por una baja densidad de hallazgos. El sustrato está compuesto por una matriz arenosa, con carbones de diferentes tamaños, raíces pequeñas, grava y gravillas en bajas proporciones. Se observaron sectores cementados debido a la presencia de altos niveles de carbonato de calcio que precipitan desde el nivel 1 hasta el nivel 3 (B. Ercolano, com pers.). Si bien la cementación observada fue mayor en el nivel 2 -entre los 1,20 a 1,17 m-, el carbonato de calcio se registró a lo largo de toda la estratigrafía hasta 1,30 m de pro- fundidad, presentándose como concreciones adheridas a huesos y moluscos y en forma de pequeñas esferas dispersas desde el sector superior hasta los niveles inferiores del depósito. La presencia de carbonato de calcio, bajo la forma de pequeños nódulos o recubriendo restos arqueológicos que se observa en el perfil, es de origen pedogénico (Gile et al., 1966), y estaría vinculada a la disolución de los restos de moluscos, la precipitación in situ y un probable ascenso capilar (Birkeland, 1999) (B. Ercolano, com pers.).

\section{METODOLOGÍA}

La metodología de estudio involucró el análisis tafonómico de las distintas clases de evidencia recuperadas, a saber, restos de malacofauna, de vertebrados y piezas líticas. Los restos malacológicos fueron identificados taxonómicamente a nivel de especie, género o familia a partir de caracteres morfológicos y taxonómicos utilizados para la identificación según la nomenclatura de Aguirre \& Farinati (1999) y la base de datos del 
Sistema de Información Taxonómica Integrado (ITIS). La cuantificación tomó en cuenta el estado de conservación [valvas o conchas enteras (VE), cuando conservaban más del $90 \%$ de la pieza y un elemento diagnóstico individual o Elemento No Repetitivo (NRE); y Fragmento identificable (FI)]. Los fragmentos con menos del $90 \%$ conservado pero que tenían presente el NRE fueron considerados como especímenes identificables (Manson et al., 1998). La cuantificación se basó en el Número de Fragmentos Identificables (NISP) y el Número Mínimo de Individuos (MNI) para cada género o especie. En el caso de bivalvos el MNI tuvo en cuenta la lateralidad. Para estimar el tamaño de las valvas de Mytilus edulis se aplicaron técnicas morfométricas, que consisten en la aplicación de análisis estadísticos que permiten determinar la relación existente entre las longitudes de diferentes partes de los moluscos (largo, ancho, largo del umbo, inserción muscular, etc.), y a partir de las ecuaciones derivadas del ajuste que presenten estas relaciones, estimar la longitud total de los individuos. Se consideraron para ello el largo y ancho de la valva, el largo del ligamento (Claassen, 1998: 109, fig. 20) y el largo del umbo. Se realizaron pruebas de normalidad y homogeneidad de varianza y regresiones lineales para determinar los parámetros morfométricos. Una vez calculados los parámetros se procedió a estimar la longitud total de los individuos fragmentados, aplicando ecuaciones morfométricas obtenidas a partir de las muestras control.

La preservación del conjunto arqueomalacológico consideró, además de la articulación y fragmentación (grado 1: $>90 \%$, grado 2: entre el $90-50 \%$ y grado 3: $<50 \%$ ), la abrasión externa (Brett \& Baird, 1986; Parsons, 1991; Gordillo et al., 2010), borde chipeado, preservación del color original (Claassen, 1998), la preservación de periostraco, de encostramiento (Fernández López, 1999) y de sedimento. Dentro de las modificaciones que tienen lugar antes de la muerte del individuo (bioerosión) se distinguió la perforación, la incrustación y las marcas de golpe.

El conjunto de vertebrados fue identificado a nivel taxonómico (NISP) y anatómico (MNE). Las modificaciones óseas consideradas incluyeron el estado de meteorización, abrasión, fracturas, daños por carnívoros, por pisoteo, marcas antrópicas y de raíces, concreciones de carbonato y alteración térmica; modificaciones que fueron relevadas macroscópicamente por medio de lupas de mano de entre $3 x$ y $15 x$. Para la meteorización subaérea seguimos la escala propuesta por Behrensmeyer (1978) para restos óseos de mamíferos de un peso mayor a $5 \mathrm{~kg}$, así como también perfiles que surgen de la agrupación de estos estadios. Éstos últimos son «fresco» (estadio 0), «levemente meteorizado» (estadios 1 y 2) y «significativamente meteorizado» (estadios 3 a 5). Las restantes variables mencionadas fueron tomadas de la bibliografía (ver Lyman, 1994; Muñoz, 2008).

En el análisis tafonómico del conjunto lítico se consideraron las modificaciones producidas por la abrasión eólica (corrasión) en contextos costeros (Borrazzo, 2006). Se tuvo en cuenta la localización (presencia en una o dos caras de la pieza) y la intensidad (grado de desarrollo de la modificación sobre cada pieza), siguiendo los estadios propuestos por Hiscock (1985) y adaptados por Borrazzo (2006). La intensidad de la abrasión considera cuatro estadios desde piezas frescas (sin abrasión) hasta piezas completamente abradidas. Para ello se analizaron todas las piezas a excepción de las lascas primarias, ya que estas últimas, por poseer una cara con reserva total de corteza, no permiten observar claramente el desarrollo de abrasión sobre dicha superficie. También se registraron la presencia de precipitados químicos (revestimiento de rocas sensu Turner, 1972) y las modificaciones por raíces. Todas las observaciones se realizaron macroscópicamente y mediante el empleo de lupa de mano de $30 X$.

\section{RESULTADOS}

\section{Análisis Tafonómico Del Conjunto Malacológico}

Los 206 especímenes recuperados fueron asignables a siete especies de los cuales $92,18 \%$ corresponden a bivalvos y $7,82 \%$ a gasterópodos. Entre los bivalvos se observa un predominio de la familia Mytilidae con tres especies Aulacomya atra, Brachidontes purpuratus y Mytilus edulis. En cuanto a la composición del sitio predominan $A$. atra $(46,96 \%)$, seguida por M. edulis $(24,35 \%) \mathrm{y}$ B. purpuratus $(20,87 \%)$. Los gasterópodos, representados por cuatro especies, se hallan presentes en muy bajos porcentajes.

La proporción de alteraciones registradas en los restos malacológicos asignables a bioerosión (producidas antes de su recolección) es muy baja 


\begin{tabular}{|c|c|c|c|c|c|c|c|c|c|}
\hline & \multirow{2}{*}{\multicolumn{2}{|c|}{ Variables cuantificadas }} & \multicolumn{3}{|c|}{ Bivalvia } & \multicolumn{4}{|c|}{ Gasterópodos } \\
\hline & & & Mytilus edulis & $\begin{array}{l}\text { Aulacomya } \\
\text { atra }\end{array}$ & B. purpuratus & Fisurella sp. & $\begin{array}{c}\text { Iothia } \\
\text { coppingeri }\end{array}$ & Nacella sp. & $\begin{array}{l}\text { Buccinanops } \\
\text { sp. }\end{array}$ \\
\hline & NISP & & 49 & 75 & 40 & 1 & 1 & 4 & 3 \\
\hline \multirow{9}{*}{ 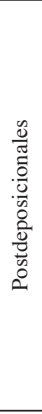 } & \multirow{4}{*}{$\frac{\overline{0}}{0}$} & Color original & - & - & - & - & - & - & - \\
\hline & & $\begin{array}{l}\text { Preservación } \\
\text { parcial }\end{array}$ & $26(53,06 \%)$ & $24(32 \%)$ & $10(25 \%)$ & - & - & - & - \\
\hline & & Pérdida total & $23(46,94 \%)$ & $51(68 \%)$ & $30(75 \%)$ & $1(100 \%)$ & $1(100 \%)$ & $4(100 \%)$ & $3(100 \%)$ \\
\hline & & $\begin{array}{l}\text { Pérdida por } \\
\text { calcinación }\end{array}$ & - & - & - & - & - & - & - \\
\hline & \multirow{8}{*}{ 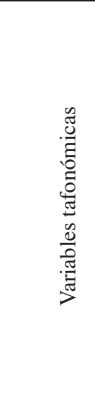 } & $\begin{array}{l}\text { Preservación del } \\
\text { periostraco }\end{array}$ & - & - & - & - & - & - & - \\
\hline & & Abrasión & $35(71 \%)$ & $33(44 \%)$ & $12(30 \%)$ & - & - & - & - \\
\hline & & Encrostamiento & $37(75,5)$ & $59(79 \%)$ & $23(57,9 \%)$ & - & - & $2(50 \%)$ & $3(100 \%)$ \\
\hline & & Borde chipiado & $44(89,8 \%)$ & $70(93,33 \%)$ & $29(72,5 \%)$ & $1(100 \%)$ & $1(100 \%)$ & $2(50 \%)$ & $3(100 \%)$ \\
\hline & & Cementación & $5(10,20 \%)$ & $1(1,33 \%)$ & - & - & - & - & - \\
\hline \multirow{3}{*}{ 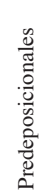 } & & Perforación & $2(4,08 \%)$ & $4(5,41 \%)$ & $2(5 \%)$ & - & - & - & - \\
\hline & & Incrustación & $2(4,08 \%)$ & $4(5,41 \%)$ & $2(5 \%)$ & - & - & - & - \\
\hline & & Marcas de golpe & - & $4(5,33 \%)$ & $3(7,89 \%)$ & - & - & - & $1(100 \%)$ \\
\hline
\end{tabular}

TABLA 1

Modificaciones pre- y pos- deposicionales en el conjunto malacológico recuperado.

en todo el conjunto. No se observó incrustación en ninguno de los especímenes y la presencia de perforaciones no supera el $6 \%$ en todos los casos. Lo mismo ocurre con las marcas de golpe que solo están presentes en menos del 10\% de los especímenes.

En lo que hace a las modificaciones post-deposicionales el total del material analizado se encontró desarticulado, siendo la proporción de valvas izquierdas y derechas similar para cada una de las especies (ver Caracotche et al., 2017: tabla 2). En cuanto a la fragmentación se registra una variabilidad entre las especies. La mejor conservada es $B$. purpuratus registrando más del $60 \%$ de sus valvas enteras. Tanto M. edulis como A. atra presentan un bajo estado de conservación con más del $70 \%$ de los especímenes fragmentados. Sin embargo, se observan diferencias entre estas dos especies, $M$. edulis presenta mejor estado de conservación con un $34,69 \%$ de valvas en grado 1 frente a un $18,67 \%$ de A. atra.

La fragmentación que presentan los restos de bivalvos afecta la estimación del tamaño de las valvas recolectadas. Si se considera M. edulis, las diferencias en cuanto a los tamaños que componen el conjunto difiere según se consideren la totalidad de la muestra (VE + FI) o solo las piezas enteras (VE). La estimación a partir solo de las VE muestran predominancia de valvas de 5 y $4 \mathrm{~cm}$, ambos tamaños con una representación de 33,3 \% cada una; sin embargo al considerar tanto las VE y las FI predominan tres tamaños por igual, las valvas de 4, 5 y $6 \mathrm{~cm}$ con un $21,6 \%$ (Figura 3 ).

Otra variable analizada fue la abrasión externa. Comparativamente las valvas de M. edulis fueron las que mayor desgaste sufrieron con más del $70 \%$ de sus valvas con signo de abrasión, seguida por $A$. atra y siendo la mejor conservada $B$. purpuratus. A nivel de bordes en todas las especies se observa más del $70 \%$ chipeado y la más afectada es $A$. atra. El $100 \%$ de los especímenes presenta perdida de color original y de periostraco. Entre los mitílidos, $B$. purpuratus presenta mayor pérdida de color con más del $70 \%$ de sus valvas afectadas, seguido por

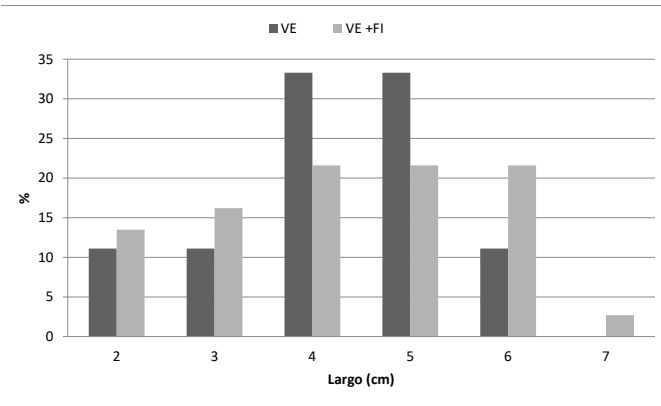

FIGURA 3

Comparación de los rangos de tamaño medidos en VE y estimados por morfometría FI en el conjunto de M. edulis. 
A. atra con más del $60 \%$ y M. edulis menos del $50 \%$ de valvas.

Tanto M. edulis como A. atra presentan del $75 \%$ de las valvas con encostramiento, mientras que $B$. purpuratus eel $57.9 \%$. La cementación pudo registrarse en sólo 5 valvas de $M$. edulis y en una de $A$. atra.

\section{Análisis Tafonómico Del Conjunto De Vertebrados}

El conjunto está formado por fragmentos de pequeño tamaño, que en el caso de los identificados a nivel específico, presentan abundantes marcas de raíces (Tabla 2). Las concreciones de carbonato de calcio en la superficie de estos materiales alcanzan a solo tres especímenes, por lo que no representan un sesgo respecto de las posibilidades de reconocer modificaciones óseas en éstos materiales. Los fragmentos con señales de combustión suman a 7 especímenes (Tabla 2) y ningun especimen registró evidencias de abrasión.

En lo que respecta a la composición taxonómica de los 158 restos óseos recuperados 71 son indeterminados, correspondiendo los restantes a Lama guanicoe (7\%), pinnípedos (15\%), aves indeterminadas $(19 \%)$, Rhea pennata $(7 \%)$ y peces $(39 \%)$ (Tabla 2). Por otro lado, estos restos corresponden a elementos anatómicos de distintos tamaños, edades ontogenéticas y densidad mineral ósea, por lo que se trata de un conjunto diverso no sólo en relación al tamaño de los restos sino también en lo que hace a la procedencia de los mismos (animales marinos y terrestres) y su resistencia diferencial a los procesos tafonómicos.
Los restos de las distintas especies presentan claras marcas de origen antrópico, lo que indica una intervención humana en la formación del depósito y en la depositación de los restos de los distintos taxones (ver Tabla 2).

En lo que hace a la historia deposicional, un aspecto a calibrar es la resolución e integridad (sen$s u$ Binford, 1981) que presenta este conjunto. Los restos de vertebrados ofrecen información sensible en este sentido, particularmente a partir del estado de meteorización de los materiales. En la Figura 4 puede apreciarse que los restos de los distintos taxones muestran varios estadios representados, ocupando el estadio 1 un lugar dominante en esta distribución. Esta representación se corresponde con perfiles levemente meteorizados, en los subconjuntos de guanaco, pinnípedos y aves, y en menor proporción restos en estado fresco (pinnípedos $\mathrm{y}$ aves, ver Figura 5).

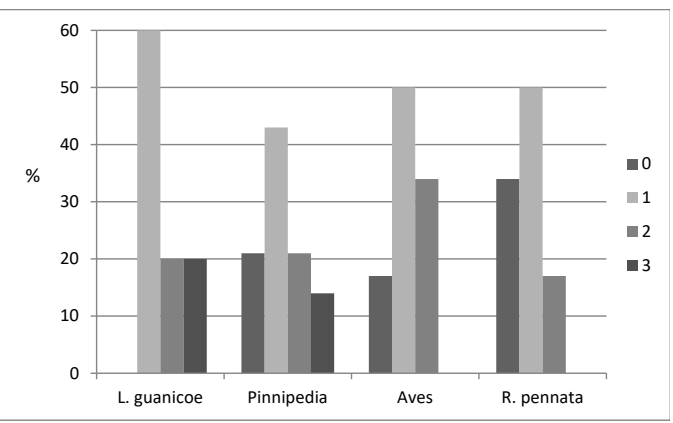

FIGURA 4

Estadios de meteorización relevados en los restos de los distintos vertebrados recuperados en YQ3. La escala numérica corresponde a cada estadio (0 a 5).

\begin{tabular}{|c|c|c|c|c|c|c|c|c|c|}
\hline & \multirow[b]{2}{*}{ NSP } & \multirow[b]{2}{*}{ NISP } & \multirow[b]{2}{*}{ MNE } & \multicolumn{3}{|c|}{ NISP con huellas antrópicas } & \multirow[b]{2}{*}{ raíces } & \multirow[b]{2}{*}{$\mathrm{CaCO}_{3}$} & \multirow[b]{2}{*}{ quemado } \\
\hline & & & & corte & machacado & co+ma & & & \\
\hline Vertebrados indeterminados & 35 & & & & & & 1 & & \\
\hline Mamífero indeterminado & 36 & & & & & & & 2 & 4 \\
\hline L. guanicoe & & 6 & 6 & 2 & & & 4 & & \\
\hline Pinnipedia & & 14 & 10 & 1 & & & 7 & 1 & \\
\hline Rodentia & & 6 & 2 & & & & & & 1 \\
\hline Ctenomys sp. & & 4 & 3 & & & & & & \\
\hline Aves & & 16 & 7 & 2 & 1 & 1 & 7 & & 1 \\
\hline R.pennata & & 6 & 4 & 2 & & & 5 & & \\
\hline Pisces & & 34 & 33 & & & & & & 1 \\
\hline Merluccius sp. & & 1 & 1 & & & & & & \\
\hline Total & 71 & 87 & 66 & 7 & 1 & 1 & 24 & 3 & 7 \\
\hline
\end{tabular}

TABLA 2

Modificaciones pre- y pos- deposicionales en el conjunto de vertebrados recuperado. 


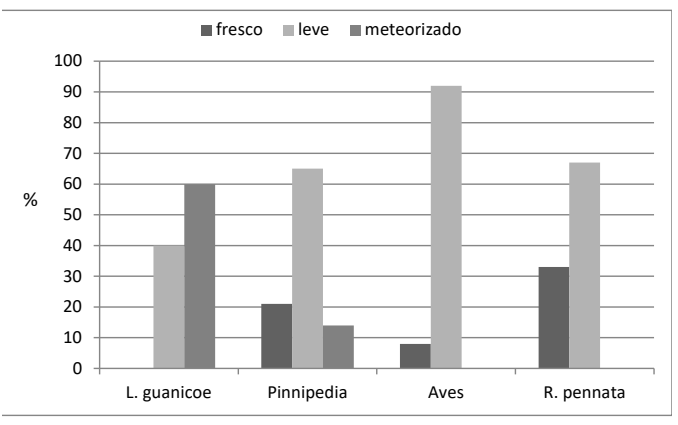

FIGURA 5

Perfiles de meteorización que surgen de la agrupación de los estadios de meteorización presentados en la Figura 4.

\section{Análisis Tafonómico Del Conjunto Artefactual Lítico}

De los 147 artefactos que componen la muestra, 132 registran evidencias de abrasión eólica y 104 presentan revestimiento de carbonato de calcio. La mayor parte de los artefactos abradidos tienen ambas caras alteradas, es decir, el efecto de la abrasión se registra sobre toda la pieza, lo cual implica que la misma fue volteándose de modo tal que se vio expuesta una y otra cara a lo largo del tiempo. La muestra no presenta raíces adheridas a pesar de provenir de un contexto estratigráfico. Esto podría relacionarse con la ausencia de vegetación en el sector donde se encontraba contenido el material.

Se registraron todos los grados de abrasión pero el de mayor importancia es el 1, el cual consiste en una abrasión del tipo suave. Le siguen en importancia, aunque en una proporción mucho menor, el 2 (abradido) y por último el 3 (muy abradido) (Figura 6). Cuando se compara el grado de abrasión por materia prima se observa que ésta afectó de modo similar a todas las rocas. Existen sutiles diferencias pero que pueden estar mediadas por el tamaño de la muestra; por ejemplo, la calcedonia no registra abrasión. Si bien se trata de una roca más dura, este dato debe tomarse con precaución ya que sólo hay 3 piezas de esta materia prima en todo el registro. A partir de estas observaciones puede afirmarse que este conjunto responde a una misma historia tafonómica. En cuanto a la precipitación de carbonato de calcio, se observó que la mayor parte de la muestra se encuentra afectada por este fenómeno. Al igual que en el caso anterior, la mayoría de los artefactos registra esta alteración en sus dos caras.
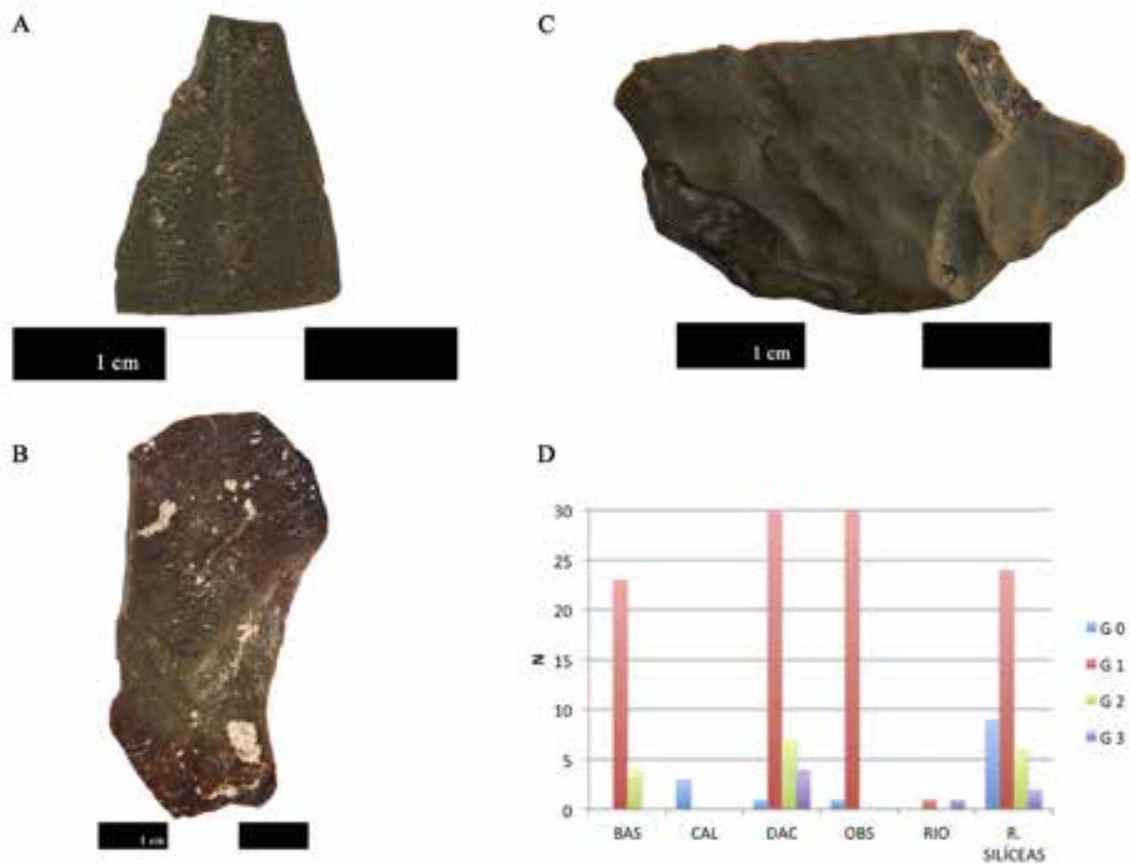

FIGURA 6

Abrasión eólica registrada en los materiales líticos de YQ3. A = Grado 1, B = Grado 2, C = Grado 3. Distribución de las materias primas por grado de abrasión: Bas (basalto), CAL (calcedonia), DAC (dacita), OBS (obsidiana), RIO (riolita), R. Silíceas (rocas silíceas). G 0 (grado 0), G 1 (grado 1), G 2 (grado 2), G 3 (grado 3). 


\section{DISCUSIÓN}

Los distintos indicadores considerados muestran una historia tafonómica en la que tanto la exposición subaérea como el enterramiento tuvieron lugar en la formación de este sitio arqueológico. Varios de ellos sugieren que se trata de una asociación de materiales con relativa integridad, como surge de la presencia de pares de valvas, perfiles de meteorización semejantes en restos de distintos vertebrados, y grados de abrasión leves e intermedios en los restos de malacofauna y artefactos líticos. Es importante mencionar, como se señaló, que los restos de mamíferos y aves presentan marcas antrópicas.

Los atributos tafonómicos que más afectaron el conjunto arqueomalacológico fueron la fragmentación, la perdida de color, la abrasión externa y el encostramiento. Los procesos de fragmentación y abrasión externa fueron variables en las diferentes especies. Las diferencias en este sentido podrían deberse a las características de la microestructura y grosor estas valvas que responden de manera diferencial a los diferentes agentes del ambiente. La resistencia a la fragmentación de las estructuras calcáreas está determinada también por las características propias de la valvas como la morfología, la microestructura, el grosor, la ornamentación, el tamaño y la resistencia de la valva (Aguirre et al., 2011; Hammond, 2015). Del análisis de las longitudes de los individuos de Mytilus edulis antes y después de la aplicación de las ecuaciones morfométricas comprobamos que la fragmentación afectó a todos los tamaños de valvas. Las longitudes obtenidas a partir de analizar solo las valvas enteras o enteras y fragmentadas mostraron diferencias longitudinales del conjunto, de esta manera comprobamos que la fragmentación genera sesgos en la estimación de la longitud al sobredimensionar algunos tamaños (e.g. 4 y $5 \mathrm{~cm}$ ) sobre otros (ver Figura 3). Si consideramos que solo el $30 \%$ del material se encontraba completo, la fragmentación afecta tanto la interpretación tafonómica como los análisis que a partir de estos materiales se haga, por ejemplo, el cálculo del aporte calórico que estos moluscos pueden brindar por individuo (ver Lobbia, 2016). Por lo tanto la morfometría constituye una herramienta de gran utilidad que para el análisis e interpretación del material malacológico.

Con respecto a la preservación del color, ninguna valva presenta color original y en todas las es- pecies más del $40 \%$ lo ha perdido totalmente. Sin embargo, este indicador de exposición en ningún caso estuvo asociado a calcinación solar, lo que es indicativo de que las valvas nunca estuvieron expuestas de manera prolongada en el tiempo en la superficie. Sin embargo, la pérdida del color original de las valvas está vinculada no solo a la exposición al sol sino que se relaciona especialmente con la ocurrencia de otros procesos tafonómicos como la abrasión, la corrosión y la alteración térmica (Hammond, 2015). Por lo tanto, considerando el alto porcentaje de abrasión podría pensarse que la perdida de color estuvo más bien vinculada con este proceso que con prolongadas exposiciones al sol. Por otra parte, se observó ausencia total de periostraco en todas las muestras. Según algunos autores, como Evans (1972) y Boborwski (1984), entre otros, el periostraco se destruye rápidamente al estar expuesto a las condiciones medioambientales fuera del agua, por lo que una alta exposición en la superficie podría favorecer la rápida pérdida de dicha capa y su conservación estaría vinculada con un rápido enterramiento $(\mathrm{Zu}-$ bimendi, 2012; Hammond \& Zubimendi, 2013). Sin embargo, aún en condiciones de rápido enterramiento y en contextos estatigráficos, dicha capa se ve afectada por las características del sedimento como el $\mathrm{pH}$, la proporción de materia orgánica y la humedad (Hammond, 2014). Por último, todas las valvas presentan un alto porcentaje de encostramiento, alta presencia de sedimento pero baja cementación. Esto estaría indicando la ocurrencia de un proceso de mineralización debido probablemente a una sobresaturación de agua del depósito. Sin embargo, este proceso no fue lo suficientemente prolongado o intenso para manifestarse en la cementación de cavidades. El análisis de la malacofauna muestra que las diferencias en los distintos grados de fragmentación, color y preservación de la capa prismática de las distintas especies identificadas puede deberse a diferencias en la composición química de las valvas representadas en el conjunto. Sin embargo, en todos los casos podemos inferir una exposición subaérea corta y enterramiento prolongado.

En lo que tiene que ver con el conjunto de restos de vertebrados, los distintos taxones muestran una tendencia similar en lo que a la representación de los estadios de meteorización se refiere, que abarca del estadio cero al tres, y que indica que el conjunto pasó por una etapa de exposición que habría afectado de manera similar a los materiales. El 
subconjunto guanaco se distingue en parte de ésta tendencia ya que presenta una proporción mayor de restos meteorizados (estadio 3) y ausencia de estadio 0. Asimismo se trata del subconjunto con una mayor variación entre la meteorización mínima y máxima relevada en cada espécimen, lo que sugiere una historia depositacional más compleja o prolongada, respecto de los otros subconjuntos. En este sentido no está claro si esa diferencia podría estar vinculada a distintos aportes de restos en el tiempo. En efecto, la presencia de marcas antrópicas y los distintos grados de meteorización se encuentran en restos de los niveles inferiores como superiores, por lo que no hay una asociación clara entre grado de meteorización y ausencia de marcas de procesamiento y nivel estratigráfico (Tabla 2). Aunque los restos de aves forman un subconjunto en el que no está representado el grado «meteorizado» su origen depositacional está igualmente asociado al comportamiento humano, como surge del $18 \%$ de restos con marcas de éste origen. El subconjunto de restos de pinnípedos, por su parte, presenta una situación intermedia entre el de guanacos y el de aves (Figura 5). Por otro lado, las evidencias vinculadas a la etapa de enterramiento en el conjunto de restos de vertebrados incluyen, principalmente, las improntas dejadas por las raíces de la vegetación, presentes en el $26 \%$ de los restos identificados taxonómicamente, entre ellos los correspondientes a guanaco, pinnípedos, rheidos y aves indeterminadas, y en menor medida los que presentan depositación de carbonato de calcio (Tabla 2).

El análisis de las distintas variables relevadas en los restos artefactuales líticos también informan de un conjunto dinámico que pasó por etapas de exposición subaérea y otras de enterramiento, siendo probablemente ésta la más larga dada la baja incidencia de la abrasión eólica en relación a la datación del conjunto (si bien hay muchos artefactos abradidos, la mayoría presenta una abrasión suave lo cual resulta llamativo si se considera que el depósito se formó hace 5.000 años). Esta idea se refuerza con lo observado para los moluscos, que no presentan pérdida de color producto de la calcinación solar y, por ende, de un tiempo prolongado de exposición en superficie. Por otra parte, la presencia del revestimiento de carbonato de calcio sugiere que el conjunto lítico estuvo enterrado hasta el momento de su descubrimiento, ya que de otro modo el mismo hubiese sido eliminado por la intervención de factores medioambientales como la lluvia y como consecuencia de la abrasión generada por la acción del viento en conjunto con los granos de arena sueltos y disponibles en el ambiente. La presencia de marcas de raíces en los restos óseos y la ausencia de raíces adheridas a los restos líticos estaría indicando que la cobertura vegetal pudo ser más importante en el pasado que en el presente, al momento de recuperación el sector donde se encontraba contenido el material presentaba ausencia de vegetación. Esta transición de condiciones de estabilidad, vinculadas al enterramiento y desarrollo de cobertura vegetal, a condiciones de exposición o re-exposición deja señales disímiles en las distintas clases de evidencia y contribuye a una evaluación mas completa de los depósitos bajo estudio (ver por ej. Cañete Mastrángelo \& Muñoz, 2018).

En síntesis, el análisis tafonómico de las distintas clases de evidencia consideradas en el sitio arqueológico YQ3 permite postular que presenta suficiente integridad como para sostener que se trata de un depósito antropogénico. La historia tafonómica, de más de 5000 años, permite inferir que el conjunto pasó por momentos de exposición y enterramiento. Si bien las etapas vinculadas a la exposición subaérea pudieron ser importantes, éstas no fueron dominantes en la conformación del conjunto a juzgar por el modo en que los materiales con distinta resistencia diferencial están representados y reflejan la acción de los distintos procesos de deterioro.

\section{CONCLUSIONES}

La evaluación de las características tafonómicas de depósitos antiguos, como YQ3, formado por diversas clases de evidencia permite avanzar sobre las características que hacen al registro arqueológico de las ocupaciones humanas en la costa patagónica durante el Holoceno medio. El análisis de múltiples líneas de evidencia está orientado a entender el rango de variación de este registro y, como se señaló más arriba y en otra publicación (Caracotche et al., 2017), este objetivo es particularmente relevante para un bloque temporal en el que la evidencia no solo es escasa sino limitada, en relación a la información potencial que los depósitos ofrecen. El análisis aquí efectuado procuró desarrollar y articular el análisis de clases de evidencia con resistencia diferencial a 
los elementos ambientales muy distinta. De esta manera fue posible evaluar las evidencias de exposición (abrasión, meteorización, etc.) en conjunto con las señales de integridad (pares de valvas representados, edades ontogenéticas, densidad mineral ósea, etc.). La conclusión es que se trata de un depósito con señales claras de acumulación a partir de actividades humanas, que presenta una relativa integridad en la asociación de materiales recuperados. Desde un punto de vista tafonómico destaca el aporte que las técnicas de análisis morfométricos ofrecen para calibrar la composición del conjunto arqueomalacológico. Asimismo, el análisis del impacto de la abrasión sobre materiales tan distintos como las valvas de moluscos y los artefactos líticos permitió enriquecer la discusión sobre la intensidad de acción de los procesos destructivos.

El registro arqueológico de YQ3 amplía el rango de información sobre el modo de utilización de la costa patagónica en el Holoceno medio. Muestra que se aprovecharon recursos provenientes de ambientes diversos (terrestres y marinos), que involucraron una diversidad de comportamientos de captura (ver Caracotche et al., 2017). Si bien esta evidencia está de acuerdo con los datos conocidos para las ocupaciones costeras del Holoceno medio de la costa de Santa Cruz, el análisis aquí efectuado permite ampliar la discusión sobre la naturaleza de estos registros y la importancia relativa de los distintos procesos que intervienen en su formación y transformación a lo largo del tiempo.

\section{AGRADECIMIENTOS}

Este trabajo es un resultado de los proyectos PIP/CONICET 112-201201-00359, UNPA 29/ A364 y SECyT UNC RES. 313/16. Autorización de investigación APN No 804. Agradecemos a todo el personal del Parque Nacional Monte León que nos asistió en la logística y tareas de campo. Los Guardaparques Mariana Martínez, Pablo Contreras, Cristian Vellido y los brigadistas Diego Brescia, Leonardo Martínez y Rubén Sosa junto a Gustavo Nauto colaboraron en las tareas de campo. Agradecemos a Fabiana Cantarell (SIB-APN) por su ayuda en la realización de la Figura 1.

\section{REFERENCIAS}

Aguirre, M.L. \& Farinati, E.A. 1999: Taphonomic processes affecting late quaternary molluscs along the coastal area of Buenos Aires province, Argentina, Southwestern Atlantic. Paleogeography, Paleoclimatology, Paleoecology 149: 283-304.

Aguirre, M.L.; Richiano, S.; Farinati, E. \& Fucks, E. 2011: Taphonomic comparison between two bivalves (Mactra and Brachidontes) from Late Quaternary deposits in northern Argentina: Which intrinsic and extrinsic factors prevail under different palaeoenvironmental conditions?. Quaternary International 233(2): 113-129.

Behrensmeyer, A.K. 1978: Taphonomic and ecologic information from bone weathering. Paleobiology 4(2): 150-162.

BINFORD, L. 1981: Bones: Ancient Men and Modern Myths. Academic Press, New York.

Birkeland, P.W. 1999: Soils and geomorphology. $3^{\text {rd }}$ ed. Oxford University Press, Oxford.

Bobrowsky, P.T. 1984: The History and Science of Gastropods in Archaeology. American Antiquity 49 (1): 77-93.

Borrazzo, K. 2006: Tafonomía lítica en dunas: una propuesta para el análisis de los artefactos líticos. Intersecciones en Antropología 7: 247-261.

Brett, C. \& BAIRD, G. 1986: Comparative taphonomy: a key to paleoenvironmental interpretation based on fossil preservation. Palaios 1: 207-227.

Cañete Mastrángelo, D.S. \& Muñoz, A.S. 2018: Tafonomía de depósitos en superficie: el caso de P 37 (desembocadura Del Río Santa Cruz), Patagonia Meridional. Arqueología 24(2): 161-176.

Caracotche, M.S.; Cruz Espinosa, I.S.; Carballo, F. \& Belardi, J.B. 2005: Rescate arqueológico en el Parque Nacional Monte León (Santa Cruz, Argentina). Magallania 33(2): 143-163.

Caracotche, M.S.; Muñoz, A.S.; Cañete, D.S. \& LobBIA, P.A. 2017: El registro arqueológico del Holoceno medio en el Parque Nacional Monte León (Patagonia, Argentina). Magallania 45(2):165-180.

Castro, A.; Moreno, J.; Zubimendi, M.; Blanca Videla, M.A.; Ambrústolo, P.; Mazzitelli, L. \& Bogan, S. 2007: Cronología de las ocupaciones humanas de la costa norte de Santa Cruz. En: Morello, F.; Martinic, M.; Prieto, A. \& Bahamonde, G. (eds): Arqueología de Fuego-Patagonia. Levantando piedras, desenterrando hueso y develando arcanos: 527-539). Ediciones CEQUA, Punta Arenas.

ClaAssen, C.P. 1998: Shells (Manuals in Archaeology). Cambridge University Press, Cambridge. 
Cruz, I.; Muñoz, A.S. \& Lobbia, P. 2011: La explotación de recursos marinos en la costa de Patagonia continental: los restos de vertebrados en depósitos de Punta Entrada y Monte León (Santa Cruz, Argentina). Revista de Estudios Marítimos y Sociales 4: 31-41.

Evans, J.W. 1972: Tidal growth increments in the cockle Clinocardium nuttalli. Science 176(4033): 416-417.

Fernández López, S. 1999: Tafonomía y Fosilización. En: Meléndez, B. (ed.): Tratado de Paleontología: 51-107, 438-441. CSIC, Madrid.

Gile, L.; Peterson, F.F. \& Grossman, R.B. 1966: Morphological and genetic sequences of carbonate accumulation in desert soils. Soil Science 101: 347-360.

Gordillo, S.; Bayer, M.S. \& Martinelli, J. 2010: Moluscos recientes del Canal Beagle, Tierra del Fuego: un análisis cualitativo y cuantitativo de Los ensambles de valvas fósiles y actuales. Anales Instituto $\mathrm{Pa}$ tagonia 38(2): 95-106.

Hammond, H. 2014: Taphonomic analysis of archaeomalacological assemblages: shell middens on the northern coast of Santa Cruz (Patagonia, Argentina). Intersecciones en Antropología 15: 21-34.

- 2015: Sitios concheros en la costa norte de Santa Cruz: su estructura arqueológica y variabilidad espacial en cazadores recolectores patagónicos. Tesis de Doctorado. Facultad de Ciencias Naturales y Museo, Universidad Nacional de La Plata, La Plata. Ms.

Hammond, H. \& Zubimendi, M.A. 2013: Estudio de la composición de sitios concheros en la Costa Norte de Santa Cruz (Patagonia Argentina). En: Zangrando, F.; Barberena, R.; Gil, A.; Neme, G.; Giardina, M.; Luna, L.; Otaola, C.; Paulides, S.; Salgán, L. \& Tívoli, A. (eds.): Tendencias teórico metodológicas y casos de estudio en la Arqueología de la Patagonia: 405-415. Altuna impresores, Buenos Aires.

Hiscock, P. 1985: The need for a taphonomic perspective in stone artefact analysis. Queensland Archaeological Research 2: 82-95.

LoBbia, P. 2016: Análisis morfométrico en Mytilus edulis: una herramienta para el análisis de los sitios con- cheros y la arqueología de la costa. Revista Arqueología 22: 141-165.

Lyman, R. 1994: Vertebrate Taphonomy. Cambridge University Press, Cambridge.

Mason, R.; Peterson, M. \& Tiffany, J. 1998: Weighing vs. counting: measurement reliability and the California school of midden analysis. American Antiquity 63: 303-324.

MuÑoz, A.S. 2008: El procesamiento de los camélidos fueguinos en el pasado. Aspectos metodológicos y resultados alcanzados para el sector atlántico de Tierra del Fuego. En: Acosta, A.; Loponte, D. \& Mucciolo, L. (comps.): Temas de arqueología. Estudios Zooarqueológicos y Tafonómicos (I): 77-97). Instituto Nacional de Antropología y Pensamiento Latinoamericano, Buenos Aires.

Muñoz, A.S.; Caracotche, M.S. \& Cruz, I. 2009: Cronología de la costa al sur del río Santa Cruz: nuevas dataciones radiocarbónicas en Punta Entrada y Parque Nacional Monte León (Provincia de Santa Cruz). Magallania 37(1): 39-43.

Oliva, G.; Humano, G.; Rial, P.; González, L.; Paredes, P.; Kofalt, R.; Mascó, M.; Ferrante, D.; FranchiNI, C. \& Vivar, M.E. 2006: Estudio de línea de base y plan de monitoreo de la biodiversidad vegetal del Parque Nacional Monte León. Informe técnico. EEA Santa Cruz INTA-UNPA-CAP, Santa Cruz.

Parsons, K.M. 1991: Taphonomic processes and biases in modern marine environments: an actualistic perspective on fossil assemblage preservation. In: Donovan, S. (ed.): The Processes of Fossilization: 22-65. Columbia University Press, New York.

Turner, J.C.M. 1972: Diccionario Geológico. Asociación Geológica Argentina, Serie B Didáctica y Complementaria 71: 288.

ZuBimendi, M.A. 2012: La variabilidad del registro arqueomalacológico en la costa norte de Santa Cruz (Patagonia argentina): resultados exploratorios a partir de estudios estratigráficos. Intersecciones en Antropología 13(2): 359-375. 
